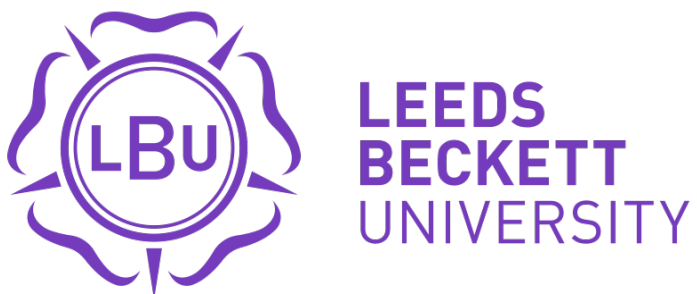

Citation:

Tipples, J and Lupton, M and George, D (2021) Speeding up time: Hierarchical Bayesian drift diffusion modelling evidence for accelerating temporal accumulation. Timing and Time Perception, 9 (4). pp. 393-416. ISSN 2213-445X DOI: https://doi.org/10.1163/22134468-bja10030

Link to Leeds Beckett Repository record:

https://eprints.leedsbeckett.ac.uk/id/eprint/7912/

Document Version:

Article (Accepted Version)

The aim of the Leeds Beckett Repository is to provide open access to our research, as required by funder policies and permitted by publishers and copyright law.

The Leeds Beckett repository holds a wide range of publications, each of which has been checked for copyright and the relevant embargo period has been applied by the Research Services team.

We operate on a standard take-down policy. If you are the author or publisher of an output and you would like it removed from the repository, please contact us and we will investigate on a case-by-case basis.

Each thesis in the repository has been cleared where necessary by the author for third party copyright. If you would like a thesis to be removed from the repository or believe there is an issue with copyright, please contact us on openaccess@leedsbeckett.ac.uk and we will investigate on a case-by-case basis. 


\title{
Speeding up Time: Hierarchical Bayesian Drift Diffusion Modelling Evidence for Accelerating Temporal Accumulation
}

\author{
Jason Tipples ${ }^{1, *}$, Michael Lupton ${ }^{2}$ and David George ${ }^{2}$ \\ ${ }^{1}$ Psychology Group, Leeds Beckett University, Leeds, UK \\ ${ }^{2}$ Department of Psychology, University of Hull, Hull, UK \\ Received 16 August 2020; in revised form 31 December 2020; accepted \#\#\#\#
}

* To whom correspondence should be addressed. E-mail: $\underline{\text { W.Tipples@leedsbeckett.ac.uk }}$ 
Speeding-up time 2

\begin{abstract}
Time perception is malleable — it can be made to speed up and slow down by various experimental manipulations including the presentation of a sequence of auditory clicks and also angry facial expressions. Recent evidence supports the idea that auditory click trains increase accumulation of evidence across time. Here, we test this idea for both angry expressions and auditory clicks by modelling response times (and choice responses) using Bayesian Hierarchical Drift Diffusion Modelling. Two separate groups of participants (Experiment 1; $n=29$; Experiment 2; $n=38$ ) judged the duration of angry and neutral facial expressions preceded by either a 3-s sequence of auditory clicks or silence. In both experiments, standard psychophysical analyses showed that both clicks and angry expressions lengthened the perception of time. The original finding came from the analyses of the Drift Diffusion Modelling parameter that represents the speed of information accumulation - the drift rate parameter. Drift rates grew in magnitude with the duration of the face and moreover this effect was larger when the faces were either preceded by clicks or appeared angry — evidence for accelerating temporal accumulation. This novel insight would not have been possible from traditional psychophysical analyses and therefore, the results highlight the potential value of Bayesian Hierarchical Drift Diffusion Modelling as a tool for understanding how we perceive time.
\end{abstract}

\title{
Keywords
}

Temporal distortion, temporal bisection. emotion, pacemaker, diffusion modelling 


\section{Introduction}

Our perception of time is easily changed. For example, studies show that people overestimate the duration of angry expressions compared to neutral facial expressions (for examples see: Bar-Haim et al., 2010; Droit-Volet et al., 2004; Effron et al., 2006; Gil \& Droit-Volet, 2011; Tipples, 2008, 2011; Tipples et al., 2015). Similar overestimation effects have been recorded for stimuli following a sequence of both auditory clicks (Droit-Volet, 2003, 2010) and flickering visual stimuli (e.g., Droit-Volet \& Wearden, 2002; Ortega \& López, 2008). The novelty of the current research is to apply Drift Diffusion Modelling to time perception (Balc1 \& Simen, 2014; Simen et al., 2011, 2013) to help understand how auditory clicks and angry expressions affect time perception.

The temporal bisection task (Church \& Deluty, 1977; Wearden, 1991) is frequently used to examine the effects of auditory clicks and angry expressions on time perception. In the temporal bisection task, participants first learn to recognise short and long durations and then during a second stage, decide whether test stimulus is closer in duration to either the short or long duration they learnt earlier. The test stimuli (e.g., simple shapes or faces) are presented for a range of durations including the short and long durations. Researchers often calculated and analyse two indices of timing called the Bisection Point and Weber Ratio. The Bisection Point is the comparison duration giving rise to $50 \%$ of the long responses - lower values show that participants start responding long sooner. The Weber Ratio is a standardised index of temporal sensitivity - lower values indicate higher sensitivity. In brief, studies have shown that participants respond long more often and the bisection point is lower for both angry expressions compared to neutral facial expressions (Droit-Volet et al., 2004) and also, lower following a sequence of auditory clicks compared to silence (e.g., Droit-Volet, 2010). Evidence for increased temporal sensitivity (a lower Weber Ratio value) is less consistent across studies for both auditory clicks and angry expressions. In short, time is overestimated following clicks and angry expressions.

How do clicks and angry expressions affect time perception? One explanation is that they increase arousal and the consequently the speed of an arousal-sensitive pacemaker mechanism that resides within a dedicated internal clock (Gibbon et al., 1984; Rammsayer \& Ulrich, 2001; Treisman, 1963; Treisman et al., 1990; Zakay \& Block, 1997). Recently, this idea has been challenged by the results of studies in which the authors recorded similar overestimation effects for both clicks (Droit-Volet, 2010; Jones et al., 2011) and angry 
expressions (Droit-Volet, 2013; Young \& Cordes, 2013) when participants judged nontemporal quantities.

Indeed, the results of one study that compared the effect of clicks on the judgement of time, length and number (Droit-Volet, 2010) supports the idea that clicks lead to a general accumulation of evidence across time. Specifically, a temporal bisection task was used in which the to-be-timed stimuli were presented either sequentially (e.g., line length grew in size across displays) or nonsequentially (e.g., in a single display). Following previous research, Droit-Volet showed that clicks shifted the psychophysical functions and lowered the bisection points. The same effect occurred for length and number judgements following clicks but only when the stimuli were presented sequentially. Sequences are by definition changes across time and therefore, the results of the study by Droit-Volet (Droit-Volet, 2010) support the idea that auditory clicks increase the accumulation of evidence across time.

Sequential-sampling models of perceptual choice (including Ratcliff's Drift Diffusion Model; Ratcliff, 1978) model the accumulation of evidence across time by modelling RT distributions in addition to choice response data (e.g., the proportion of long responses). In other words, the Drift Diffusion Model is a further way to test the idea that auditory clicks and facial expressions increase the accumulation of evidence across time and therefore, we applied this modelling approach in the current research.

In the Drift Diffusion Model of two-alternative forced-choice decisions (Ratcliff, 1978), the operation of latent psychological processes can be inferred from four main model parameters. These parameters are the drift rate $v$, starting point $z$, boundary separation $a$, and the nondecision time parameter $T_{\text {er }}$. These processes are shown in Fig. 1. The drift rate parameter describes the speed at which evidence accumulates toward a specific decision threshold (e.g., a long response). The drift rate is modelled as following a random walk until a threshold is reached and a decision is made. In tasks that record accuracy, fast accurate responses are indicative of a high drift rate - fast information accumulation. The boundary separation parameter is an index of the amount of information required for a decision. Smaller boundary separation values indicate liberal and perhaps impulsive response criteria (less information is required for a decision). Large boundary values indicate conservative response criteria (more information is required for a decision). The starting point parameter $z$ represents the starting point of the drift diffusion process. If the starting point parameter increases in one condition above 0.5 then this may represent a response bias. For example, punishing participants for failing to classify a stimulus from a specific category might result in a higher starting point for classifying stimuli as belonging to that category (irrespective of the actual 
stimulus presented on a specific trial). The nondecision parameter models all processes occurring before or after the decision, for example encoding processes (before) and response execution processes (after).

Recently, researchers have extended the Drift Diffusion Model to model timing data (Rivest \& Bengio, 2011; Simen et al., 2011) and more specifically, data from the temporal bisection task (Balc1 \& Simen, 2014). Specifically, the two-stage, sequential diffusion model of temporal decision making proposed by Balcı and Simen (2014) maps the latent cognitive processes described in the Drift Diffusion Model to two distinct stages. First, during the to-betimed stimulus presentation, temporal information is predicted to accumulate following a random walk toward a single long threshold. During this stage the starting point of the drift diffusion process is initially set to be closer to the short threshold based on the assumption that a short response will be preferred until sufficient time elapses. As time lengthens the decision to respond long is strengthened (based on the accumulation of evidence) and consequently, the starting point parameter moves toward the long decision boundary. The second process starts immediately after the offset of the interval (or timed stimulus). During the second stage, the time estimate from the first stage — indexed by the starting point parameter — is compared to bisection criteria - midway between the short and long threshold. Participants respond long if the difference exceeds the long threshold and respond short if the difference exceeds the short threshold.

Recently, we used a Bayesian Hierarchical version of the Drift Diffusion Model (Vandekerckhove et al., 2011; Wiecki et al., 2013) to test for increased accumulation due to fear and also to test the main predictions of the two-stage process described by Balc1 and Simen (2014). Specifically, high and low fearful groups judged the duration of spider and bird images in a temporal bisection. The results support the main predictions of the model described by Balc1 and Simen and also offered clear support for increased temporal accumulation due to fear — drift rates were high for spiders compared to birds in high but not low spider-fearful participants. Also, the starting parameter — the starting point of the drift diffusion process was initially higher for spiders in high fearful individuals - an effect attributed to the rapid accumulation of time information during the initial presentation of the spider images.

\subsection{Objectives of the Present Study}

Here, the main aim was to model data from the temporal bisection task using Drift Diffusion Modelling to test the idea that clicks and angry expressions increase the accumulation of 
evidence across time. To test this idea we used a fully within-subjects design in which participants judged the duration of both angry and neutral facial expressions that were preceded equally often by either a 3-s sequence of auditory clicks or silence. An advantage of a withinsubjects design is that it is possible to account for individual differences by using the appropriate error term and therefore, this design has both greater statistical precision compared to a between-subjects design and also avoids making inferences based on the nonstatistical comparison of effects across experiments. One concern might be that the inclusion of multiple conditions could increase experimental noise making it difficult to replicate previous research. To pre-empt, the current research replicates previous findings in addition to providing new information from the results of Drift Diffusion Model.

For analyses of the drift diffusion parameter the key question was BalciDo click trains and angry expressions increase drift rates?Balc1 and also, BalciDoes the magnitude of the drift rate difference for click trains and angry expressions increase with stimulus duration?Balc1 The latter question is relevant because if the effects of clicks and angry expressions affect the rate of accumulation then the effects should grow in magnitude with stimulus duration — they should accelerate.

\section{Material and Methods}

\subsection{Participants}

Twenty-nine undergraduate psychology students ( 25 female, mean age $=20 ; 4$ male, mean age $=20$ ) from the University of Hull participated in return for a course credit. All had normal or corrected-to-normal vision.

\subsection{Materials and Apparatus}

The faces were 20 digitised photographs from the NimStim set of facial expressions (Tottenham et al., 2009) of four males and five females each displaying an angry and neutral facial expression were used. When presented in the centre of the computer screen, at an average distance of $60 \mathrm{~cm}$, the faces measured $18^{\circ}$ of vertical visual angle. The auditory cue was a train of auditory clicks ( $1000 \mathrm{~Hz}$ tones) repeated for $3 \mathrm{~s}$ at $10 \mathrm{~Hz}$. The clicks were presented at approximately $65 \mathrm{~dB}$ and were delivered binaurally through Sennheiser HD 280 digital headphones. All visual stimuli were presented on a 24 -inch computer monitor $(1280 \times 1080,60 \mathrm{~Hz})$. Stimulus presentation and data collection were controlled by E-Prime software (Schneider et al., 2002). 


\subsection{Procedure}

All participants completed learning and test phases. In the learning phase, participants were trained to discriminate short $(400 \mathrm{~ms})$ from long $(1,600 \mathrm{~ms})$ stimulus durations. On the first eight trials, a pink oval appeared for either a short or long duration in a fixed sequence (e.g., long-short-long-short, etc.). Participants were told to expect this sequence and to press the $\mathrm{z}$ or $\mathrm{m}$ to indicate whether the oval appeared for either a short or long duration. The response mapping (e.g., $\mathrm{z}$ for short durations and $\mathrm{m}$ for long durations) was counterbalanced across participants. The auditory click train was not presented during the training phase. Following a response, participants were presented with visual feedback lasting $500 \mathrm{~ms}$, for both correct ('yes') and incorrect ('no') decisions. The feedback was followed by a fixed $1000 \mathrm{~ms}$ intertrial interval. In the final stage of the learning phase, the pink oval was presented for a further eight trials in a new random order for each participant. Participants continued to indicate whether the oval appeared for either short or long stimulus durations and received feedback.

During the test phase, the oval was replaced by the face stimuli. Participants were asked to (a) look at the face and (b) indicate whether the face appeared for a duration that was closer to either the short or long durations that they had learnt earlier. Feedback was not given during the main test phase. In the test phase, there were 28 possible trial types that were derived from the factorial combination of duration $(7) \times$ expression (angry, neutral) $\times$ click condition (click train, no click). The 20 faces were presented in each cell of the 2 (click condition; clicks, no clicks) $\times 7$ (duration; 400, 600, 800, 1000, 1200, 1400, $1600 \mathrm{~ms}$ ) design leading to the creation of 280 trials -10 trials for each combination of expression, click condition and duration. A new randomised trial order was created for each participant. Each trial began with a fixed $1000 \mathrm{~ms}$ blank interval. The face stimuli were displayed immediately following the offset of the auditory clicks or blank interval.

\subsection{Analyses of Bisection Points and Weber Ratios}

The Bisection Point and Weber ratio were calculated separately for each participant, expression and cue condition. The Bisection Point refers to the point of subjective equality (the 0.5 point on the psychometric function). If participants repeatedly respond 'long' sooner as the stimulus duration increases then the estimated duration at which they respond equally often short or long (the Bisection Point) will be lower, indicating an overestimation or lengthening effect. The BP for each participant in each condition was calculated from the intercept and slope parameters of the logistic regression of $p$ (long) onto stimulus duration. The software R (R Core Team, 2014) and more specifically, the $\operatorname{glm}()$ function was used to estimate the regression coefficients. The Weber Ratio is a measure of temporal sensitivity and is calculated by dividing half the difference between the upper and lower difference limen [ $p$ (long) (0.75) and $p$ (long) (0.25), respectively] by the Bisection Point.

Bayesian mixed ANOVAs were conducted on the mean BPs and WRs using the BayesFactor software in R (Morey \& Rouder, 2015). Models were created from all possible combinations of expression and click condition by including or excluding main effects or interactions with the constraint that if an interaction was included the corresponding main effects were also included (Rouder et al., 2012). Bayes factors were estimated using a noninformative Jeffreys prior on the variance of the population and a Cauchy prior with 
default scales on the standardised effect size for effects (Rouder et al., 2012). Following Jeffery's scheme, Bayes Factors for the alternative hypothesis $\left(\mathrm{BF}_{10}\right)$ were classified as either 'anecdotal' (1-3), 'moderate' (3$10)$, 'strong' (10-30), 'very strong' (30-100), or 'decisive' (>100). With respect to the null hypothesis, Bayes Factors ( $\left.\mathrm{BF}_{01}\right)$ were classified as either 'anecdotal' (1-0.33), 'moderate' (0.33-0.1), 'strong' (0.1-0.03), 'very strong' (0.03-0.01), or 'decisive' (<0.01). Differences were considered 'significant' or noteworthy if Bayes Factors values offered at least 'anecdotal' support for either the null or alternative hypothesis.

As follow-up tests of differences we used a one-sample Bayesian $t$-test to estimate both standardised effect sizes Cohen's $d_{z}(\mu-0 / \sigma)$ and the 95\% highest density interval (HDI) around the effect size difference from zero. We used the BEST package (Kruschke \& Meredith, 2017) for the latter calculations with minimally informative priors, i.e., normal priors with large standard deviation for $(\mu)$, broad uniform priors for $(\sigma)$, and a shifted-exponential prior for $(v)$. Cohen (Cohen, 1988) provided guidelines for interpreting the magnitude of effect sizes namely, Cohen's $d_{\mathrm{z}}=0.2$ (small), Cohen's $d_{\mathrm{z}}=0.5$ (medium) and Cohen's $d_{\mathrm{z}}=0.8$ (large). A region of practical equivalence (ROPE) was defined as effect sizes falling within Cohen's $d_{\mathrm{z}}$ in the range $-0.1-0.1$. A 95\% HDI that falls outside this region might be considered 'statistically significant'. If more that $5 \%$ falls within the range $-0.1-0.1$ the effect is considered as 'equivalent' (or nonsignificant).

We have placed example code for the Diffusion Model (described next) in the Appendix and placed datasets and other code on the OSF repository https://osf.io/gqcbk/?view only=6d7cbd8ae20a4ac39f20beffd426b40a)

\subsection{Hierarchical Drift Diffusion Modelling}

We estimated the drift diffusion parameters using a Hierarchical Drift Diffusion Modelling procedure (Vandekerckhove et al., 2008, 2011; Wiecki et al., 2013) programmed in Python (Wiecki et al., 2013). HDDM is a Bayesian Modelling approach that uses Markov Chain Monte Carlo (MCMC) simulations to estimate a range of probable values for diffusion parameters - posterior distributions of parameter values. Statistical inference can be made directly from comparison of overlap between posterior distributions; there is no need for aggregation and frequentist analyses. Hierarchical Bayesian estimation is suited to modelling datasets with a relatively small number of observations per cell of the design because subject and group-level posterior estimates reciprocally influence each other.

\subsection{Hierarchical Bayesian Regression}

In the model described by Balcı and Simen (2014), temporal accumulation is modelled by estimating the drift rate and starting point parameters. Therefore, in the current manuscript we tested the hypothesis that click trains and angry expressions would increase temporal accumulation by modelling changes in drift rates and, in separate models, the starting points. All other parameters (e.g., nondecision times, boundary separation values) were free to vary across individuals. 
A regression modelling approach was used. Wiecki et al. (2013) provide a useful tutorial for implementing this type of model. In all models, expression (angry, neutral) and click condition (click, no click) were entered as categorical (treatment-coded) predictors with the neutral face and no-click conditions serving as the baseline (or reference category) for each individual in each condition. Face duration (400, 600, 800, 1000, $1200,1400,1600 \mathrm{~ms}$ ) was entered into the regression equation as continuous covariate. To aid interpretation of conditional main effects (when interactions are present) we re-parameterised the duration values to $0,1,2,3,4,5$ and 6. The slope for duration is a direct test of the prediction that drift rates will increase linearly with each $200 \mathrm{~ms}$ of stimulus exposure - one of the key predictions in the model described by Balc1 and Simen (2014). Following previous research, we also modelled the starting point parameter. The prediction was that drift rates would rise more rapidly for the click and angry expression conditions (during picture presentation) leading to a higher starting point parameter for the start of the second stage decision process described by Balc1 and Simen (2014).

\subsection{Model Assessment}

All hierarchical drift diffusion regression models included random intercepts for the subjects for each of the four main diffusion parameters $(v, t, z$, and $a)$. We modelled changes in both the drift rate and starting point by estimating three regression models: a main effects model, a two-way interaction model and a full three-way interaction. The main effects model included the fixed effects of click condition (click, no click), expression (angry, neutral) and face duration (linear). The two-way interaction model included the theoretically relevant duration $\times$ expression and duration $\times$ click condition interaction terms and all and the nested, conditional main effects. The three-way interaction model included all possible interactions and the nested, conditional main effects. For analyses of the drift diffusion parameter the key statistical tests were the main effects model ('Do drift rates increase for click trains and angry expressions?') and the multiplicative model ('Do drift rates increase for click trains and angry expressions with increases in stimulus duration?'). The Deviance Information Criterion (DIC; Spiegelhalter et al., 2002) was used as criterion for assessing model fit. Although smaller DIC values indicate a better fit, the reduction needs to be sufficiently large to justify an increased complexity and therefore, we used a reduction in the DIC of 10 or more as the criterion for judging improved model fit and selecting the more complex model. Example code, details of the model convergence and model adequacy are described in the Appendix.

\section{Results}

\subsection{Bisection Point and Weber Ratio}

The mean BP and WR values for each condition are displayed in Table 1 . The mean proportion of long responses as a function of click condition, expression, and face duration are displayed in Fig 2. For the BPs a Bayesian mixed-factor ANOVA determined that the data were best represented by a model that included main effects of expression (angry, neutral) and click condition (clicks, no clicks). The Bayes factor $\left(\mathrm{BF}_{10}\right)$ was $1.34 \times 10^{13}$, 
indicating decisive evidence in favour of the main effects model when compared to the null model. The data offered little support for including the additional expression $\times$ click condition interaction term over the best-fitting model with only the main effects of expression and click condition $\left(\mathrm{BF}_{10}=0.29\right)$.

Bayesian $t$-tests showed that the BP was reached sooner for angry $(M=977, \mathrm{SD}=$ 165) compared to neutral expressions ( $M=1013, \mathrm{SD}=168)$, Cohen's $d_{\mathrm{z}}=0.63,95 \%$ HDI $[0.20,1.05]$ and, for clicks $(M=926, \mathrm{SD}=153)$ compared to no clicks $(M=1064, \mathrm{SD}=$ 152), Cohen's $d_{\mathrm{z}}=1.47,95 \%$ HDI $[0.932,2.08]$.

An identical Bayesian mixed-factor ANOVA was also performed on the Weber ratios (WR). Bayes factors favoured the null model compared to both the model with only the two main effects $\left(\mathrm{BF}_{01}=15\right)$ and the model with the two main effects and the interaction term $\left(\mathrm{BF}_{01}=45\right)$. In other words, there was little evidence for an effect of either click condition or expression on temporal sensitivity.

\subsection{Reaction Times}

For RTs (in seconds), the best-fitting model with decisive support relative to the null model included the three main effects and no interaction effects $\left(\mathrm{BF}_{10}=5.31 \times 10^{65}\right)$. There was anecdotal support for this model $\left(\mathrm{BF}_{10}=1.08\right)$ relative to the evidence for the second favourite model $\left(\mathrm{BF}_{10}=4.90 \times 10^{65}\right)$ that included only the main effects for click condition and duration. Bayesian $t$-tests revealed faster RTs for angry $(M=0.68, \mathrm{SD}=0.43)$ compared to neutral $(M=0.71, \mathrm{SD}=0.48)$ expressions, Cohen's $d_{\mathrm{z}}=0.75,95 \%$ HDI [0.27, 1.23]. RTs were also faster in the click condition $(M=0.68, \mathrm{SD}=0.49)$ compared to the noclick condition $(M=0.71, \mathrm{SD}=0.42)$, although over $10 \%$ of the HDI fell outside the ROPE and therefore was deemed not significant, Cohen's $d_{z}=0.33,95 \%$ HDI $[-0.04,0.73]$. The main effect of duration showed that RTs followed a quadratic pattern across durations; relative to the short duration, RTs increased as the decision became harder but then became faster as the duration lengthened beyond $1000 \mathrm{~ms}(400 \mathrm{~ms}=0.60 ; 600 \mathrm{~ms}=0.71$; $800 \mathrm{~ms}=0.82 ; 1000 \mathrm{~ms}=0.79 ; 1200 \mathrm{~ms}=0.73 ; 1400 \mathrm{~ms}=0.64 ; 1600 \mathrm{~ms}=0.56$ ).

\subsection{Hierarchical Drift Diffusion Modelling}

\subsubsection{Drift Rate Model Selection}


For the drift rate regression, adding the duration $\times$ expression and duration $\times$ click condition interaction terms to the main effects model improved model fit $(\mathrm{DIC}=9000)$ compared to the model with only the main effects (DIC $=9024)$. Adding the three-way interaction term failed to improve model fit $(\mathrm{DIC}=9001)$ and therefore, the model with the duration $\times$ expression and duration $\times$ click interaction terms was used as the basis for statistical inference.

\subsubsection{Drift Rates}

Regression analyses of the linear trend for face duration for neutral faces in the no-click condition supports the key prediction of the model proposed by Balc1 and Simen (2014); for every $200 \mathrm{~ms}$ there was a strong increase in drift rates $[\beta=0.57 ; 95 \%$ Bayesian credibility intervals $(\mathrm{CrI})=0.54-0.60]$.

The significant two-way interactions between both emotion and duration $(\beta=0.04 ; 95 \%$ $\mathrm{CrI}=0.01-0.07)$ and also click condition and duration $(\beta=0.07 ; 95 \% \mathrm{CrI}=0.04-0.10)$ were both positive in sign and showed that the magnitude of the effect of click condition and angry expression effects on drift rates increased with duration of the face. For faces presented for 400 $\mathrm{ms}$, drift rates increased in the click condition compared to the no-click condition $(\beta=0.17$; $95 \% \mathrm{CrI}=0.06-0.28)$. Facial expression type had no effect on drift rates when the faces were displayed for $400 \mathrm{~ms}$ - the $95 \%$ Bayesian credibility interval included the value $0(\beta=-0.05$; $95 \% \mathrm{CrI}=-0.15-0.05$. We probed the interaction by estimating the slopes (drift rate difference) for the Angry vs Neutral and Click vs No-click contrasts in the $1600 \mathrm{~ms}$ face duration condition. As shown in Fig. 3, the drift rate difference for both the Angry vs Neutral and Click vs No-click contrasts increased from 400 to $1600 \mathrm{~ms}$ and moreover, for faces presented for $1600 \mathrm{~ms}$ drift rates were higher for angry compared to neutral expressions $(\beta=0.22 ; 95 \% \mathrm{CrI}=$ $0.10-0.33)$ and also higher in the click condition compared to the no-click condition $(\beta=0.60$; 95\% CrI $=0.49-0.71)$.

\subsubsection{Starting Point Model Selection}

For the starting point regression, the main effects only model $(\mathrm{DIC}=11,146)$ provided the best fit compared both the two-way interaction model $(\mathrm{DIC}=12,512)$ and the three-way interaction model (DIC $=12,514)$ and therefore, we analysed the main effects model for the starting parameter.

\subsubsection{Starting Point Parameter}


For the starting point parameter regression (main effects) model, the results support the key prediction of the model proposed by Balc1 and Simen (2014): the starting point parameter increased with increased stimulus duration. Specifically, $95 \%$ of the probability density mass for the slope for an estimated $200 \mathrm{~ms}$ increase in duration did not contain zero $(\beta=0.06 ; 95 \%$ $\mathrm{CrI}=0.064-0.069)$. The starting parameter was also higher in the click condition compared to the no-click condition $(\beta=0.04 ; 95 \% \mathrm{CrI}=0.03-0.05)$. The $95 \%$ credibility interval for the difference between angry and neutral expressions contained the value zero $(\beta=0.002 ; 95 \% \mathrm{CrI}$ $=-0.007-0.012)$ and therefore, the results show that it is unlikely that the starting parameter differs between angry and neutral expressions.

\section{Experiment 2 - Replication Using a Different Stimulus Set}

A growing concern in psychology is that effects do not replicate (Open Science Collaboration, 2015). Experiment 2 was an attempt to establish whether the modelling results reported in Experiment 1 generalise to a different set of face stimuli. Specifically, the face stimuli used in Experiment 1 were replaced with faces that were created (using commercially available software; Poser 5 Curious Labs Inc., Santa Cruz, CA, USA) to appear angry and neutral. In previous research (Tipples, 2011) in which participants judged the duration of these face stimuli the overestimation effect for angry compared to neutral expressions was replicated. The results closely match those reported in Experiment 1 and therefore, the results are discussed together in section 7, General Discussion.

\section{Material and Methods}

Thirty-six undergraduate psychology students (35 females, mean age $=21.16$; $\mathrm{SD}=4.80 ; 3$ males, mean age = 21.13; $\mathrm{SD}=4.67$ ) from the University of Hull participated in return for a course credit. All had normal or corrected-to-normal vision and 32 were right-handed.

The method was identical to Experiment 1 except that there were fewer observations for each cell of the design. Each angry and neutral face was presented a total of five times for each cell of the click condition (click, no click $) \times$ face duration $(400,600,800,1000,1200,1400,1600 \mathrm{~ms})$ cell of the design. Two participants completed an extra block of trials due to a programming error resulting in six observations for each cell of the design.

\section{Results}


The modelling procedures and data analyses of Bisection Points and Weber Ratios were identical to those used in Experiment 1 . As noted below, the results are very similar to those reported in Experiment 1 and therefore, the results of Experiment 1 and 2 are discussed together in section 7, General Discussion.

\subsection{Bisection Points and Weber Ratio}

The mean proportion of long responses as a function of click condition, expression, and face duration for Experiment 2 are displayed in Fig 4. Following Experiment 1, for the BPs a Bayesian mixed-factor ANOVA determined that the data were best represented by a model that included main effects of expression (angry, neutral) and click condition (clicks, no clicks). The Bayes factor $\left(\mathrm{BF}_{10}\right)$ was $29,864,524$, indicating decisive evidence in favour of the main effects model when compared to the null model. The data offered little support for including the additional expression $\times$ click condition interaction term relative to the best-fitting model with the main effects of expression and click condition $\left(\mathrm{BF}_{10}=0.23\right)$.

Bayesian $t$-tests showed that the BP was reached sooner for angry $(M=1093, \mathrm{SD}=$ 138) compared to neutral expressions $(M=1158, \mathrm{SD}=169)$, Cohen's $d_{\mathrm{z}}=0.76,95 \%$ HDI $[0.36,1.18]$ and, for the click condition $(M=1079, \mathrm{SD}=153)$ compared to the no-click condition $(M=1172, \mathrm{SD}=153)$, Cohen's $d_{\mathrm{z}}=0.91,95 \%$ HDI $[0.50,1.31]$.

An identical Bayesian mixed-factor ANOVA was also performed on the WR. The best-fitting model included the main effects of expression and click condition as well as the expression $\times$ click condition $\left(\mathrm{BF}_{10}=2663\right)$. In contrast to Experiment 1 , there was moderate support for the latter model compared to models stripped of this effect $\left(\mathrm{BF}_{\text {inclusion }}=7.357\right)$.

In the click condition, the Weber Ratio was higher - indicating lower temporal sensitivity - for angry expressions $(M=0.25, \mathrm{SD}=0.07)$ compared to neutral expressions $(M=0.22, \mathrm{SD}=0.007)$, Cohen's $d_{\mathrm{z}}=-0.46,95 \%$ HDI [-0.87, -0.08$]$. In the no-click condition, although the WR was lower for angry $(M=0.19, \mathrm{SD}=0.05)$ compared to neutral $(M=0.21, \mathrm{SD}=0.007)$ expressions, more than $30 \%$ of the HDI fell within the Region of Practical Equivalence (Cohen's $d_{\mathrm{z}}=0.17,95 \%$ HDI $[-0.19,0.51]$ ) suggestive of relative equivalence between conditions. Overall, WR differences were small in magnitude. 


\subsection{Reaction Times}

For RTs (in seconds), the best-fitting model with decisive support relative to the null model included the three main effects and no interaction effects $\left(\mathrm{BF}_{10}=1.23 \times 10^{50}\right)$. There was strong support for this model $\left(\mathrm{BF}_{10}=22\right)$ relative to the evidence for the second favourite model $\left(\mathrm{BF}_{10}=5.53 \times 10^{48}\right)$ that included the additional expression $\times$ click condition interaction effect. Bayesian $t$-tests revealed faster RTs for angry $(M=0.60, \mathrm{SD}=$ $0.39)$ compared to neutral $(M=0.64, \mathrm{SD}=0.41)$ expressions, Cohen's $d_{\mathrm{z}}=0.77,95 \%$ HDI $[0.33,1.20]$ and faster RTs in the click condition $(M=0.60, \mathrm{SD}=0.41)$ compared to the noclick condition $(M=0.63, \mathrm{SD}=0.39)$, Cohen's $d_{\mathrm{z}}=0.70,95 \%$ HDI $[0.23,1.15]$. The main effect of duration showed that RTs followed a quadratic pattern across durations relative to the short duration, RTs increased as the decision became harder but then became faster as the duration lengthened beyond $1000 \mathrm{~ms}(400 \mathrm{~ms}=0.57 ; 600 \mathrm{~ms}=0.63$; $800 \mathrm{~ms}=0.72 ; 1000 \mathrm{~ms}=0.71 ; 1200 \mathrm{~ms}=0.64 ; 1400 \mathrm{~ms}=0.56 ; 1600 \mathrm{~ms}=0.49)$.

\subsection{Hierarchical Drift Diffusion Modelling}

For the drift rate regression, adding the duration $\times$ expression and duration $\times$ click condition interaction terms to the main effects model improved model fit $(\mathrm{DIC}=2907)$ compared to the model with only the main effects (DIC $=3710)$. Adding the three-way interaction term failed to improve model fit $(\mathrm{DIC}=2938)$ and therefore, the model with the duration $\times$ expression and duration $\times$ click interaction terms was used as the basis for statistical inference (Fig. 5).

\subsubsection{Drift Rates}

Following Experiment 1, regression analyses of the linear trend for face duration for neutral faces in the no-click condition supported the key prediction of the model proposed by Balc1 and Simen (2014); for every additional $200 \mathrm{~ms}$ the face was displayed for, there was a strong increase in drift rates $(\beta=0.64 ; 95 \% \mathrm{CrI}=0.61-0.68)$. The significant two-way interactions between both expression and duration $(\beta=0.12 ; 95 \% \mathrm{CrI}=0.07-0.16)$ and click condition and duration $(\beta=0.06 ; 95 \% \mathrm{CrI}=0.01-0.10)$ also followed the pattern of results reported in Experiment 1. 
The significant two-way interactions between expression and duration $(\beta=0.04 ; 95 \%$ $\mathrm{CrI}=0.01-0.07)$ and also click condition and duration $(\beta=0.07 ; 95 \% \mathrm{CrI}=0.04-0.10)$ were both positive in sign and showed that the magnitude of the effect of click condition and angry expression effects on drift rates increased with the duration of the face. For faces presented for $400 \mathrm{~ms}$, drift rates increased in the click condition compared to the no-click condition $(\beta=0.17$; $95 \% \mathrm{CrI}=0.06-0.28)$. Conversely, drift rates were lower for angry compared to neutral expressions when the faces were displayed for $400 \mathrm{~ms}$ - the $95 \%$ Bayesian credibility interval was negative and did not include the value $0(\beta=-0.18 ; 95 \% \mathrm{CrI}=-0.33--0.04)$. Following Experiment 1, we probed the interaction by estimating the slopes (drift rate difference) for the Angry vs Neutral and Click vs No-click contrasts in the 1600-ms face duration condition. As shown in Fig. 5, the drift rate difference for both contrasts increased from 400 to $1600 \mathrm{~ms}$ and moreover, for faces presented for $1600 \mathrm{~ms}$ drift rates were higher for angry compared to neutral expressions $(\beta=0.53 ; 95 \% \mathrm{CrI}=0.37-0.68)$ and also higher in the click condition compared to the no-click condition $(\beta=0.50 ; 95 \% \mathrm{CrI}=0.34-0.67)$.

\subsection{Starting Point Model Selection}

For the starting point regression, the main effects only model $(\mathrm{DIC}=5353)$ provided the best fit compared both the two-way interaction model $(\mathrm{DIC}=5355)$ and the three-way interaction model $(\mathrm{DIC}=5937)$ and therefore, we analysed the main effects model for the starting parameter.

\subsubsection{Starting Point Parameter}

For the starting point parameter regression (main effects) model, the results support the key prediction of the model proposed by Balcı and Simen (2014): the starting point parameter increased with increased face duration. Specifically, $95 \%$ of the probability density mass for the slope for an estimated $200 \mathrm{~ms}$ increase in duration did not contain zero $(\beta=0.06 ; 95 \% \mathrm{CrI}=$ 0.064-0.070). The starting parameter was also higher in the click condition compared to the noclick condition, $(\beta=0.02 ; 95 \% \mathrm{CrI}=0.01-0.03)$. The $95 \%$ credibility interval for the difference between angry and neutral expressions contained the value zero $(\beta=0.006 ; 95 \% \mathrm{CrI}=-0.005-$ 0.018 ) and therefore, the results show that it is unlikely that the starting parameter differs between angry and neutral expressions. 


\section{General Discussion}

Bayesian hierarchical Drift Diffusion Modelling provided clear support for the hypothesis that clicks and angry expressions increase the accumulation of evidence across time. This was evident from analyses of the model parameter that represents the speed of information accumulation - the drift rate parameter. Drift rates were higher when the face was preceded by a 3-s sequence of clicks (compared to no clicks). This effect grew in magnitude with the duration of the face - it became stronger with time. Drift rates were also higher for angry compared to neutral expressions although this effect was qualified by an interaction with face duration. The interaction showed that for angry expressions, the increase in drift rates took time to emerge. The specific conclusion that the accumulation of evidence accelerates with time when the faces were either angry or preceded by clicks would not have been possible from traditional psychophysical analyses. Therefore, this research highlights the value of Hierarchical Drift Diffusion Modelling as a tool for understanding how we perceive time.

The pattern of results supports a recent two-stage, sequential diffusion model of temporal bisection performance described by Balc1 and Simen (2014). In keeping with the model there was a linear increase in both drift rates and starting parameter as a function of face duration. This result is expected because the model predicts accumulation toward the long decision boundary during picture presentation leading to an increase in the drift rate with stimulus duration and a higher starting point for the second stage of the decision process. The modelling results also extend the results of a recent study (Tipples, 2015) that reported an increase in the speed of evidence accumulation for pictures of spiders (vs birds) in high, but not low spider-fearful individuals. Here, we show that increases in evidence accumulation are also found for angry facial expressions and auditory clicks.

In addition to similarities to previous findings, there are also a number of noteworthy differences. First, in previous research (Tipples, 2015) the starting parameter was higher for spider compared to bird images in spider-fearful individuals whereas in the current research, there was no effect of expression type on the starting point parameter. Second, for the angry expressions but not for the spiders used previously, the drift rate took time to emerge - the effect grew as a function of face duration. One simple explanation for the differences is that spider images but not angry expressions produce a rapid increase in arousal and this is reflected in an increase in both drift rates and the starting parameter. Put differently, the interpretation we are proposing is that for angry expressions (but not for spiders) arousal increases slowly (as the 
participant stares at the angry face) and consequently, the increase in evidence accumulation also increases slowly.

Relative differences in arousal can also help explain the observed differences between the effects of clicks and angry expressions on both the drift rate and starting point parameters. The effect of clicks on the drift rate was larger than the effect of angry expressions (see for example Fig. 2). Moreover, clicks increased the drift rate at the 400-ms face duration in a similar manner to effects recorded previously for spiders (vs birds) for high spider-fearful individuals. The clicks were presented before the to-be-timed stimulus and therefore, it makes sense that the effect of clicks on drift rates emerges more rapidly than the drift rate for angry expressions. The clicks have already been presented (before the face) and therefore, they can exert an effect across all face durations. However, the nonadditive interaction between duration and click condition shows that clicks continued to exert an effect even after they stop, suggesting a change in the rate of evidence accumulation with time - an idea we will now discuss.

One interpretation of the current results is that clicks and angry expressions speed the rate of a pacemaker mechanism that resides within an internal clock. In support, increases in the rate of evidence accumulation due to clicks and angry expressions grew with time. This is direct support for the idea that emotion and clicks affect the rate of an internal mechanism such as a pacemaker. Indeed, one might question whether Drift Diffusion Modelling is really necessary given that existing pacemaker-accumulators can be extended to produce a model that makes similar predictions to the model reported here (for details, see Simen et al., 2013). The reason for choosing Drift Diffusion Modelling is pragmatic: In previous work (Tipples et al., 2015) using the temporal bisection task, RTs in addition to choice responses (the proportion of long responses) were affected by the presentation of emotional facial expressions and consequently we wanted a way of incorporating the analysis of RTs with choice proportion data. Drift Diffusion Modelling does this and produces model parameters that have a psychological meaning such as the drift rate as an index of evidence accumulation. Moreover, the Drift Diffusion Modelling used here - Hierarchical Drift Diffusion Modelling — is particularly useful because, as a Bayesian approach, it has particular statistical strengths when the number of observations per cell of the design is low. Finally, Drift Diffusion Modelling is a general model of two-alternative forced-choice decision making. It is possible to use it and remain agnostic about the existence of any specific representations (e.g., time units). This is an advantage for modelling the effects of auditory clicks and facial expressions because recent research (e.g., Droit-Volet, 2010, 2013; Young \& Cordes, 2013) has shown that both stimuli 
types produce overestimation effects for nontemporal decisions - the effects are not specific to time perception.

One criticism that might be levelled at this study is that it used a complex design that added noise to the data collection. This conclusion is not supported by the results. Analyses of the Bisection Point and Weber Ratio replicate previous studies of the effects of auditory click trains and angry expressions on time estimates. Specifically, in support of the conclusion that participants perceived time as longer following angry expressions and click trains, Bisection Points were lower following clicks (compared to silence) and lower for angry compared to neutral expressions. There was little support for reduced temporal sensitivity for either angry expressions (vs neutral expressions) or for responses in the click compared to the no-click condition. An unexpected interaction effect reported in Experiment 2 was moderate in size and not in keeping with the idea of increased noise due to either hearing clicks or seeing angry expressions. Overall, analyses of the Bisection Points support the conclusions reached in previous research that people perceive time as lasting longer when the to-be-timed event is either an angry-looking facial expression or is preceded by a sequence of clicks.

\section{Future Work}

Future research might attempt to combine Drift Diffusion Modelling with the time course of neural activity recorded using EEG. This will allow researchers to move beyond mapping statistical effects to neural activity to mapping latent cognitive process (represented by the parameters of Drift Diffusion Modelling) to neural activity. Recent research (Kononowicz \& van Rijn, 2014) on this topic has shown that individual differences in the subjective experience of time correlate with relatively early neural activity (N1P2 amplitudes) rather a later CNV component as previously hypothesised (Macar et al., 1999). If N1P2 amplitude indexes the neural generator responsible for temporal accumulation (or receives output from such a generator) then N1P2 amplitude during time estimation should be larger for clicks and angry expressions relative to their control conditions.

\section{Conclusion}

The Diffusion Model results reported here corroborate previous research using psychophysical methods by showing that click trains and angry expressions increase the speed of information accumulation - they accelerate temporal accumulation. 
Speeding-up time 19 


\section{Appendix}

\section{Example Code for Experiment 1 - Best-fitting Drift Rate Model}

import pandas as pd

import matplotlib.pyplot as plt

import hddm

import kabuki

get_ipython().magic(u'matplotlib inline')

data $=$ hddm.load_csv('Nim_Click_HDDM.csv')

TwoWayDriftModel = hddm.HDDMRegressor(data, " $\sim$ C(Expression,

Treatment('neutral'))+C(SoundFile, Treatment('silence') $)+$ StndDur+C(Expression, Treatment('neutral')):StndDur+C(SoundFile, Treatment('silence')):StndDur", bias=True, include='z')

TwoWayDriftModel.find_starting_values()

TwoWayDriftModel.sample(5000, burn=200, thin=3, dbname='traces.db', db='pickle')

TwoWayDriftModel.save(' TwoWayDriftModel')

TwoWayDriftModel.plot_posteriors()

\section{Model Estimation and Convergence}

Hierarchical Drift Diffusion Modelling is a Bayesian statistical approach and therefore, it is necessary to specify priors. Following the recommendations for HDDM (Wiecki et al., 2013) we used informative priors to constrain parameter estimates to be within the range of plausible values estimated in previous research (Matzke \& Wagenmakers, 2009).

Each model was estimated without assuming dependence between the drift rate $v$, and the initial bias in the starting point parameter, $z$. To assess convergence, we calculated the Gelman-Rubin convergence statistic and also carried out visual inspection (for each estimated parameter) of three plots: (1) trace, (2) autocorrelation, and (3) posterior distribution. The Gelman-Rubin statistic requires multiple MCMC runs to estimate the ratio of between-chain variance relative to within-chain variance. Chain stability is indicated by values close $(+/-0.01)$ to 1 . For all models, we calculated the Gelman-Rubin statistic by running four MCMC chains composed of 200 samples as a burn-in (to increase chain stability) and a subsequent 5000 iterations to estimate the posterior distribution of each parameter. For all models, the Gelman- 
Rubin statistic was close to $1(+/-0.01)$. For example, the Gelman-Rubin statistic for the expression $\times$ duration interaction for drift rates was 1.003 . For all fixed effects visual inspection of the three plots failed to reveal either (1) large jumps or asymmetry in the trace or (2) high levels ( $>0.04)$ of autocorrelation (after 50 iterations) or (3) nonnormality in the distribution of the posteriors.

To illustrate convergence, we have plotted the trace, autocorrelation and posterior distribution for a single MCMC chain for both the click condition $\times$ duration drift rate interaction (Fig. 6) and expression $\times$ duration drift rate interaction (Fig. 7) for Experiment 1.

Figure 8 shows the results of a posterior predictive check for the drift rate model for Experiment 1 - an attempt to assess the adequacy of the model by comparing fitted values to the actual data. The predicted values were estimated by drawing 200 samples from the posterior distribution of that model. The data displayed in Fig. 8 are averaged across the 200 samples and show the observed vs predicted long response RTs for the $0.1,0.3,0.5$, and 0.9 RT quantiles for the drift rate model for Experiment 1. Further plots (and the data) are available on request. 


\section{References}

Balc1, F., \& Simen, P. (2014). Decision processes in temporal discrimination. Acta Psychol., 149, 157-168. doi: 10.1016/j.actpsy.2014.03.005

Bar-Haim, Y., Kerem, A., Lamy, D., \& Zakay, D. (2010). When time slows down: The influence of threat on time perception in anxiety. Cogn. Emot, 24, 255-263. doi: 10.1080/02699930903387603

Church, R. M., \& Deluty, M. Z. (1977). Bisection of temporal intervals. J. Exp. Psychol. Anim. Behav. Proc., 3, 216-228. doi: 10.1037/0097-7403.3.3.216

Cohen, J. (1988). Statistical Power Analysis for the Behavioral Sciences. New York, NY: Routledge Academic.

Droit-Volet, S. (2003). Alerting attention and time perception in children. J. Exp. Child Psychol., 85, 372-384. doi: 10.1016/s0022-0965(03)00103-6.

Droit-Volet, S. (2010). Speeding up a master clock common to time, number and length? Behav. Proc., 85, 126134. doi: 10.1016/j.beproc.2010.06.017

Droit-Volet, S. (2013). Emotion and magnitude perception: number and length bisection. Front. Neurorobot., 7 , 24. doi: 10.3389/fnbot.2013.00024

Droit-Volet, S., \& Wearden, J. (2002). Speeding up an internal clock in children? Effects of visual flicker on subjective duration. Q. J. Exp. Psychol. B, 55, 193-211. doi: 10.1080/02724990143000252

Droit-Volet, S., Brunot, S., \& Niedenthal, P. M. (2004). Perception of the duration of emotional events. Cogn. Emot, , 18, 849-858. doi: 10.1080/02699930341000194

Effron, D. A., Niedenthal, P. M., Gil, S., \& Droit-Volet, S. (2006). Embodied temporal perception of emotion. Emotion, 6, 1-9. doi: 10.1037/1528-3542.6.1.1

Gibbon, J., Church, R. M., \& Meck, W. H. (1984). Scalar timing in memory. Ann. N. Y. Acad. Sci., 423, 52-77. doi: 10.1111/j.1749-6632.1984.tb23417.x

Gil, S., \& Droit-Volet, S. (2011). “Time flies in the presence of angry faces” $0 \ldots$ depending on the temporal task used! Acta Psychol. (Amst.), 136, 354-362. doi: 10.1016/j.actpsy2010.12.010

Jones, L. A., Allely, C. S., \& Wearden, J. H. (2011). Click trains and the rate of information processing: does “speeding up" subjective time make other psychological processes run faster? Q. J. Exp. Psychol. (Hove), 64, 363-380. doi: 10.1080/17470218.2010.502580

Kononowicz, T. W., \& van Rijn, H. (2014). Decoupling interval timing and climbing neural activity: a dissociation between CNV and N1P2 amplitudes. J. Neurosci., 34, 2931-2939. doi: 10.1523/JNEUROSCI.2523-13.2014

Kruschke, J. K., \& Meredith, M. (2017). BEST: Bayesian estimation supersedes the t-test. Retrieved from https://rdrr.io/cran/BEST/

Macar, F., Vidal, F., \& Casini, L. (1999). The supplementary motor area in motor and sensory timing: evidence from slow brain potential changes. Exp. Brain Res., 125, 271-280. doi: 10.1007/s002210050683

Matzke, D., \& Wagenmakers, E.-J. (2009). Psychological interpretation of the ex-Gaussian and shifted Wald parameters: A diffusion model analysis. Psychon. Bull. Rev., 16, 798-817. doi: 10.3758/PBR.16.5.798

Morey, R. D., \& Rouder, J. (2015). BayesFactor: Computation of Bayes Factors for Common Designs. Retrieved from https://CRAN.R-project.org/package=BayesFactor

Open Science Collaboration (2015). Estimating the reproducibility of psychological. Science, 349, aac4716. doi: $10.1126 /$ science.aac4716 
Ortega, L., \& López, F. (2008). Effects of visual flicker on subjective time in a temporal bisection task. Behav. Proc., 78, 380-386. doi: 10.1016/j.beproc.2008.02.004

Rammsayer, T., \& Ulrich, R. (2001). Counting models of temporal discrimination. Psychon. Bull. Rev., 8, 270277. doi: 10.3758/BF03196161

Ratcliff, R. (1978). A theory of memory retrieval. Psychol. Rev., 85, 59-108. doi: 10.1037/0033-295X.85.2.59

Rivest, F., \& Bengio, Y. (2011). Adaptive drift-diffusion process to learn time intervals. arXiv 1103.2382 [q-Bio]. Retrieved from http://arxiv.org/abs/1103.2382

Rouder, J. N., Morey, R. D., Speckman, P. L., \& Province, J. M. (2012). Default Bayes factors for ANOVA designs. J. Math. Psychol., 56, 356-374. doi: 10.1016/j.jmp.2012.08.001

Schneider, W., Eschman, A., and Zuccolotto, A. (2002). E-Prime: User's Guide. Pittsburgh, PA: Psychology Software Inc.

Simen, P., Balc1, F., deSouza, L., Cohen, J. D., \& Holmes, P. (2011). A model of interval timing by neural integration. J. Neurosci. , 31, 9238-9253. doi: 10.1523/JNEUROSCI.3121-10.2011

Simen, P., Rivest, F., Ludvig, E. A., Balci, F., \& Killeen, P. (2013). Timescale invariance in the pacemakeraccumulator family of timing models. Timing Time Percept., 1, 159-188. doi: 10.1163/22134468-00002018

Spiegelhalter, D. J., Best, N. G., Carlin, B. P., \& Van Der Linde, A. (2002). Bayesian measures of model complexity and fit. J. R. Stat. Soc. B, 64, 583-639. doi: 10.1111/1467-9868.00353

R Core Team (2014). R: A Language and Environment for Statistical Computing. R Foundation for Statistical Computing, Vienna, Austria. Retrieved from https://cran.r-project.org

Tipples, J. (2008). Negative emotionality influences the effects of emotion on time perception. Emotion, 8, 127131. doi: $10.1037 / 1528-3542.8 .1 .127$

Tipples, J. (2011). When time stands still: fear-specific modulation of temporal bias due to threat. Emotion, 11, 7480. doi: $10.1037 / \mathrm{a} 0022015$

Tipples, J. (2015). Rapid temporal accumulation in spider fear: evidence from hierarchical drift diffusion modelling. Emotion, 15, 742-751. doi: 10.1037/emo0000079

Tipples, J., Brattan, V., \& Johnston, P. (2015). Facial emotion modulates the neural mechanisms responsible for short interval time perception. Brain Topogr., 28, 104-112. doi: 10.1007/s10548-013-0350-6

Tottenham, N., Tanaka, J. W., Leon, A. C., McCarry, T., Nurse, M., Hare, T. A., Marcus, D. J., Westerlund, A., Casey, B. J., \& Nelson, C. (2009). The NimStim set of facial expressions: Judgments from untrained research participants. Psychiatry Res., 168, 242-249. https://doi.org/10.1016/j.psychres.2008.05.006

Treisman, M. (1963). Temporal discrimination and the indifference interval: Implications for a model of the“ internal clock". Psychol. Monogr., 77, 1-31. doi: 10.1037/h0093864.

Treisman, M., Faulkner, A., Naish, P. L., \& Brogan, D. (1990). The internal clock: evidence for a temporal oscillator underlying time perception with some estimates of its characteristic frequency. Perception, 19, 705-743. doi: 10.1068/p190705

Vandekerckhove, J., Tuerlinckx, F., \& Lee, M. (2008). A Bayesian approach to diffusion process models of decision-making. Retrieved from https://irias.kuleuven.be/handle/123456789/198884

Vandekerckhove, J., Tuerlinckx, F., \& Lee, M. D. (2011). Hierarchical diffusion models for two-choice response times. Psychol. Meth., 16, 44-62. doi: 10.1037/a0021765 
Wearden, J. H. (1991). Human performance on an analogue of an interval bisection task. Q. J. Exp. Psychol. B, 43, 59-81. doi: 10.1080/14640749108401259

Wiecki, T. V., Sofer, I., \& Frank, M. J. (2013). HDDM: Hierarchical Bayesian estimation of the Drift-Diffusion Model in Python. Front. Neuroinform., 7, 14. doi: 10.3389/fninf.2013.00014

Young, L. N., \& Cordes, S. (2013). Fewer things, lasting longer: the effects of emotion on quantity judgments. Psychol. Sci., 24, 1057-1059. doi: 10.1177/0956797612465294

Zakay, D., \& Block, R. (1997). Temporal cognition. Curr. Dir. Psychol. Sci., 6, 12-16. doi: 10.1111/14678721.ep11512604 


\section{Table 1.}

Experiment 1 - Weber Ratios and Bisection Points as a function of expression and click condition. See text for details of calculation and description of the Weber Ratio and Bisection Point. Lower WR values indicate higher temporal sensitivity. The Bisection Point is the duration at which $p($ Long $)=0.5$. Bisection Points are in milliseconds.

\begin{tabular}{lccccc}
\hline Click condition & \multicolumn{2}{c}{ Weber ratio } & & \multicolumn{2}{c}{ Bisection point } \\
\cline { 2 - 3 } \cline { 6 - 6 } & Angry & Neutral & & Angry & Neutral \\
\hline Click & 0.13 & 0.15 & & 870 & 926 \\
No click & 0.12 & 0.12 & & 953 & 1076 \\
\hline
\end{tabular}




\section{Figure Captions}

Figure 1. The diffusion model for two-choice response times (RT). The evidence accumulation process begins at a specific starting point $(z)$ and subsequently follows an average increase or decrease in drift rate $(v)$. When the accumulated evidence reaches one of the boundaries a decision is made and a response is executed. The total RT includes both the decision time and Nondecision time $\left(T_{\mathrm{er}}\right)$. Nondecision time consists of both stimulus encoding and response execution processes. The distance between the two decision boundaries or boundary separation (a) can be used as an index of response caution (larger values index greater response caution).

Figure 2. Experiment 1 - the mean proportion of long responses as a function of click condition, expression and face duration.

Figure 3. Experiment 1 - the mean posterior drift rate difference as a function of face duration (400 $\mathrm{ms}, 1600 \mathrm{~ms})$ for the contrasts Angry-Neutral and Click-No click. Error bars indicate 95\% Bayesian credibility intervals (95\% CrI).

Figure 4. Experiment 2 - the mean proportion of long responses as a function of click condition, expression and face duration.

Figure 5. Experiment 2 - the mean posterior drift rate difference as a function of face duration $(400 \mathrm{~ms}, 1600 \mathrm{~ms})$ for the contrasts Angry-Neutral and Click-No click. Error bars indicate 95\% Bayesian credibility intervals (95\% CrI).

Figure 6. Example of model convergence plots of the trace (top left), autocorrelation (bottom left) and histogram posterior distribution (right) for the click condition $\times$ duration interaction effect contrast (for Experiment 1)

Figure 7. Example of model convergence plots of the trace (top left), autocorrelation (bottom left) and histogram posterior distribution (right) for the expression $\times$ duration interaction effect contrast (for Experiment 1)

Figure 8. The observed vs predicted long response RTs (seconds) for the $0.1,0.3,0.5$, and 0.9 RT quantiles for the drift rate model for Experiment 1 